\title{
First Principles Study on Li-doped and Li,O-codoped AIN
}

\author{
Honglei WU, Zuoyan QIN, Zheng YAN, Zhenhua SUN, Baikui LI, Ruisheng ZHENG*
}

\author{
Key Laboratory of Optoelectronic Devices and Systems of Ministry of Education and Guangdong Province, College of \\ Optoelectronic Engineering, Shenzhen University, Shenzhen 518060, China
}

cross ref http://dx.doi.org/10.5755/j01.ms.25.1.19138

Received 27 September 2017; accepted 18 February 2018

\begin{abstract}
This paper focuses on the detailed investigation of the structural and electronic properties of wurtzite AlN crystals doped by $\mathrm{Li}$ with and without oxygen with the first principles calculation. All the calculations have exhibited significant structural distortions. Compared with the monoclinic doping, the oxygen codoping has improved the structure deformation and lowered the formation energy of Li dopants. The calculated electronic density of states (DOS) reveals that all doping configurations still preserve semiconductor characteristics. The states around the valence band maximum cross the Fermi level, which implies p-type doping. The induced extra levels are extremely localized and flat in Li-doped AlN while much more delocalized in oxygen codoped models. The mono-doping of Li is in general energetically unfavorable while the codoping improves the formation and makes the intercalation of Li more stable in AlN. According to the results, the codoping configuration of $\mathrm{Li}$ with $\mathrm{O}$ in $\mathrm{AlN}$ has provided a useful way of modifying the corresponding properties.
\end{abstract}

Keywords: semiconductor, AlN, codoping, first principles.

\section{INTRODUCTION}

As a wide bandgap semiconductor, AlN exhibits a direct bandgap of $6.02 \mathrm{eV}$ (at room temperature), good dielectric strength and high thermal conductivity. These excellent properties make AlN a promising material for ultraviolet (UV) optoelectronic devices such as LEDs, acoustic wave devices, Schottky diodes and UV detectors. $[1,2]$ Doping is an efficient way to modify the electronic and optical performances of AlN for such applications. It has been proved experimentally that AlN doped with different impurities has shown unusual properties [3-8].

The alkali metals have attracted much attention as dopants due to the simplicity of their free-electron-like, delocalized valence state $[9,10]$. For example, Li with low electronegativity and small ionic radius facilitates high reactivity and diverse chemical bonding in the solid state $[11,12]$. Inserting Li into the $\mathrm{Al}$ site in host AlN crystals will induce holes leading to mixed-valent systems, which are beneficial for realization of p-type semiconductors. The properties of $\mathrm{Li}$ accommodated within the doped structure are very important and interesting. It is well known that oxygen being the most common impurity in AlN crystals is very hard to be removed in the growth process. Giving that the codoping method is a possible way for p-type doping of AlN [2,13,14], we think the theoretical study of codoping with $\mathrm{Li}$ and $\mathrm{O}$ atoms in AlN will be reasonable for future experiments.

First principles calculations are widely used for investigation of the ground-state properties of numerous materials. In this paper, first principles calculations of the structural and electronic properties in Li-doped and $\mathrm{Li}, \mathrm{O}-$ codoped AlN supercells were performed by the generalized

\footnotetext{
* Corresponding author. Tel.: +86-755-26538557; fax: +86-75526538557. E-mail address: rszheng@szu.edu.cn (R. Zheng)
}

gradient approximation (GGA) together with plane-wave basis sets.

\section{METHOD OF CALCULATION}

With the WIEN2K code $[15,16]$, we predict the structural and electronic properties of doped wurtzite AlN by first principle method based on density functional theory (DFT) in the GGA. The full-potential linearized augmented plane wave (FP-LAPW) method was used to describe the interaction between ion cores and valence electrons. In this method, the unit cell is divided into nonoverlapping muffin-tin atomic spheres centered on the atoms and interstitial spaces. There are two kinds of electrons, which are core and valence electrons, respectively. The Al $\left(3 s^{2} 3 p^{1}\right), O\left(2 s^{2} 2 p^{4}\right), N\left(2 s^{2} 2 p^{3}\right)$ states and outmost shell of $\mathrm{Li}$ are treated as valence electrons. Different basis sets are used for these two types of regions. Core electrons are described by atomic wave functions while the valence electrons are expanded into spherical harmonic functions in the atomic sphere and plane waves in the interstitial space. The electronic wave functions are obtained by solving Kohn-Sham equations self consistently.

The model of AlN unit cell including two $\mathrm{Al}$ atoms and two $\mathrm{N}$ atoms are building. According to the experimental results, the lattice parameters of $a$ and $c$ are chosen to $0.311 \mathrm{~nm}$ and $0.498 \mathrm{~nm}$, respectively. The supercell $(2 \times 2 \times 2)$ is a model consisting of 32 atoms. The $\mathrm{Li}$ dopants are placed on the $\mathrm{Al}$ sites while oxygen atoms replace $\mathrm{N}$ atoms. The muffin-tin radii $\left(R_{\mathrm{MT}}\right)$ for $\mathrm{Al}$, $\mathrm{N}, \mathrm{Li}$ and $\mathrm{O}$ atoms are assumed as large as possible in a way that the spheres do not overlap. The plane wave expansion of the electronic eigenfunctions in the interstitial region is cutoff with $R_{M T} \times K_{\max }=7.0$, where $R_{\mathrm{MT}}$ is the minimum radius of the muffin-tin spheres and $K_{\max }$ is the magnitude of the largest $\mathrm{K}$ vector in the plane wave. The 
$R K_{\max }$ parameter controls the size of the basis set in the FPLAPW method. The largest vector in charge density Fourier expansion $\left(G_{\max }\right)$ is set to $12.0 \mathrm{bohr}^{-1}$. The wave functions inside the muffin-tin spheres, which are expanded into spherical harmonics, are taken up to $l_{\max }=10$. The Monkhorst-Pack $k$-mesh $(5 \times 5 \times 3)$ is performed to sample the supercell Brillouin zone (BZ) in all our calculations [15], special $k$-points are obtained and the tetrahedron method [17] is used for integrations in the irreducible Brillouin zone (IBZ). The total energy was converged to less than $0.1 \mathrm{mRy}(1.36 \mathrm{meV})$ by selfconsistent calculations.

\section{RESULTS AND DISCUSSION}

The structural improvement was realized through a full relaxation of the atomic positions and internal parameters with volume optimizations of the total energy for all the calculated systems in order to reach the stable structure configuration. During the process of geometry optimization, all atoms were fully relaxed until residual forces on individual atoms become smaller than $1 \mathrm{mRy} / \mathrm{bohr}$. The volumes were optimized with the minimum energy succeeding the atomic positions relaxations. Internal structural parameters were modified using the calculated interatomic forces to determine the equilibrium atomic positions of $\mathrm{Al}, \mathrm{N}, \mathrm{Li}$ and $\mathrm{O}$. Due to the absence of experimental data concerning Li-doped AlN, the parameters of pristine $\mathrm{AlN}$ as initial conditions are used.

As seen in Table 1, Li-N moves outward after the doping. The expansion in basal plane becomes relatively smaller as the substitution takes place in the $c$-axis direction. The heterogeneously distributed bonds of tetrahedrons in pristine AlN remain anisotropy after the doping which is important for spontaneous polarizations. The bond of $\mathrm{Li}-\mathrm{N}$ in $c$-axis direction shares a very approximate distance in $\mathrm{Li}$ and $\mathrm{Li}-\mathrm{O}$ doped AlN. At the same time, the bond length along the direction perpendicular to the $c$-axis is less affected as the codoping has extended the bonding.

In order to analyze the relative stability, we study the formation energy of a separated $\mathrm{Li}$ and a $\mathrm{Li}-\mathrm{O}$ complex in AlN supercells. The formation energy with alkali metal impurity (X) of chargestate zero in AlN is defined as $[2,13]$ :

$$
E^{f}(X)=E_{t o t}[A l N, X]-E_{t o t}[A l N, b u l k]-\sum_{i} n_{i} \mu_{i}
$$

where $E_{\text {tot }}[A l N, X]$ is the total energy derived from doped AlN supercells, and $E_{\text {tot }}[A l N$, bulk] is the total energy of pristine AlN with the equivalent supercell size. $n_{i}$ denotes the number of atoms of type $i$ (host or impurity atoms) that have been added to $\left(n_{i}>0\right)$ or removed from $\left(n_{i}<0\right)$ the supercell when impurities are intercalated. $\mu_{i}$ is the corresponding chemical potentials of these atoms. Lower formation energies imply that the atoms are doped simpler and more stable in AlN.

The results clearly show that the substitution of an individual alkali metal on the $\mathrm{Al}$ site in wurtzite $\mathrm{AlN}$ is energetically unfavorable with only one exception. The formation energies of a separated $\mathrm{Li}$ on $\mathrm{Al}$ site are $-0.82 \mathrm{eV}$ for the N-rich condition and $3.29 \mathrm{eV}$ for Al-rich condition. Thus Li doped AlN is stable in N-rich growth ambient. In contrast, corresponding values of $\mathrm{Li}-\mathrm{O}$ complex in AlN are $-4.03 \mathrm{eV}$ and $-0.51 \mathrm{eV}$ respectively. By codoping with $\mathrm{O}$, the insertion of $\mathrm{Li}$ and $\mathrm{O}$ in tetrahedral positions of AlN becomes energetically favorable regardless if they take place in Al-rich or N-rich growth conditions. A very high formation energy is generated through the $\mathrm{Li}$ doping due to the fact that two extra impurity states are induced leading to substantial repulsive interaction with the neighboring $\mathrm{N}$ ions. The defect levels and weakened repulsion were modified through the $\mathrm{O}$ incorporation. The main advantage of the codoping method is reducing the formation energy by forming various complexes. In III-IV group semiconductors, $\mathrm{O}$ incorporation has always produced a number of complexes with vacancies or dopants. [2, 18] The results of this research differ from previous works [19], more theoretical and experimental investigations are expected as the calculation of formation energy is supercell-size-dependent [20].

Fig. $1 \mathrm{a}$ and $\mathrm{b}$ show the calculated band structure of Li-doped AlN and Li,O-codoped AlN, respectively. It can be clearly observed that both of them have the direct band gaps at the $\Gamma$ point. Furthermore, the calculated electronic density of states (DOS) of AlN crystals with Li doping and Li,O-codoping were obtained (presented as Fig. 2 and Fig. 3 respectively.). It is demonstrated that the distribution of states was affected by the type of doped impurities. After the doping, the electron density changes drastically, new states are produced at the top of the valence band forcing it to shift across the Fermi level.

The peaks around the Fermi level were split with decreased amplitudes from top to bottom in Fig. 2 and Fig. 3. Moreover, Fig. 4 depicts that the DOS profiles of the doped phases are similar with slight deference. The top of the VB consists predominantly of $\mathrm{Li}$ and $\mathrm{N}$ hybridization. The peaks around the Fermi level get narrower and split, which can be attributed to the weakening bonding of $\mathrm{Li}-\mathrm{N}$ with doping induced structure deformation, as discussed in Table 1.

The bottom of $\mathrm{CB}$ constructs from $\mathrm{Al}$ and $\mathrm{N}$ ions, the affection of Li can be neglected. The p-like states of the doped $\mathrm{Li}$ atoms are fairly localized which also exist in $\mathrm{Li}$ doped AlP system [9].

Table 1.Bond lengths along and perpendicular to the c-axis in the pristine and doped AlN as well as the change ratio of bond in doped AlN systems compared with the undoped system

\begin{tabular}{|l|c|c|c|c|c|c|}
\hline \multirow{2}{*}{ Structure } & \multicolumn{2}{|c|}{ Bond along c-axis } & \multirow{2}{*}{$\begin{array}{l}\text { Rate of } \\
\text { change }\end{array}$} & \multicolumn{2}{c|}{ Bond in basal plane } & \multirow{2}{*}{$\begin{array}{l}\text { Rate of } \\
\text { change }\end{array}$} \\
& type & length, a.u. & type & length, a.u. & - \\
\hline AlN & Al-N & 3.5949 & - & Al-N & 3.5700 & $7.7 \%$ \\
\hline Li: AlN & Li-N & 3.8067 & $5.9 \%$ & Li-N & 3.8444 & $0.3 \%$ \\
\hline Li-O: AlN & Li-O & 3.8365 & $6.7 \%$ & Li-N & 3.5803 & 0.3 \\
\hline
\end{tabular}




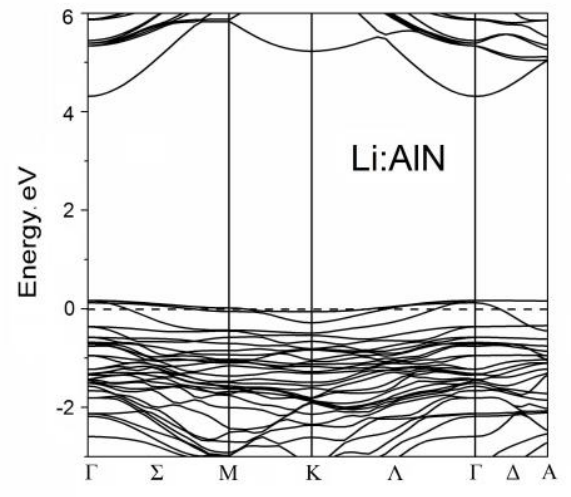

a

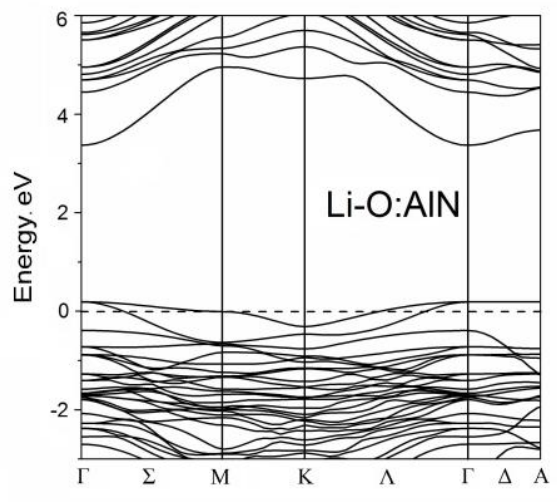

b

Fig. 1. Band structures for $a \mathrm{Li}$-doped $\mathrm{AlN}$ and $b \mathrm{Li}, \mathrm{O}$-codoped $\mathrm{AlN}$. The Fermi level is set as zero for reference

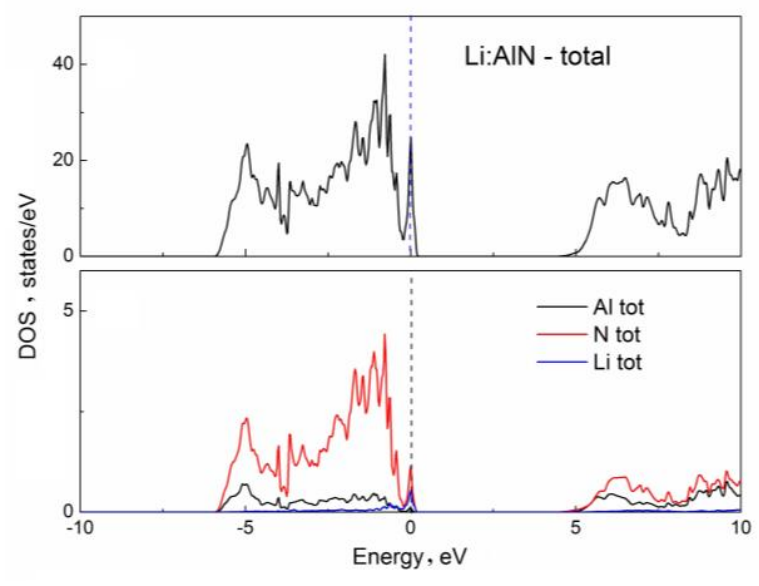

Fig. 2. DOS for Li-doped AlN: Total DOS and Partial DOS. The dashed line denotes the Fermi level

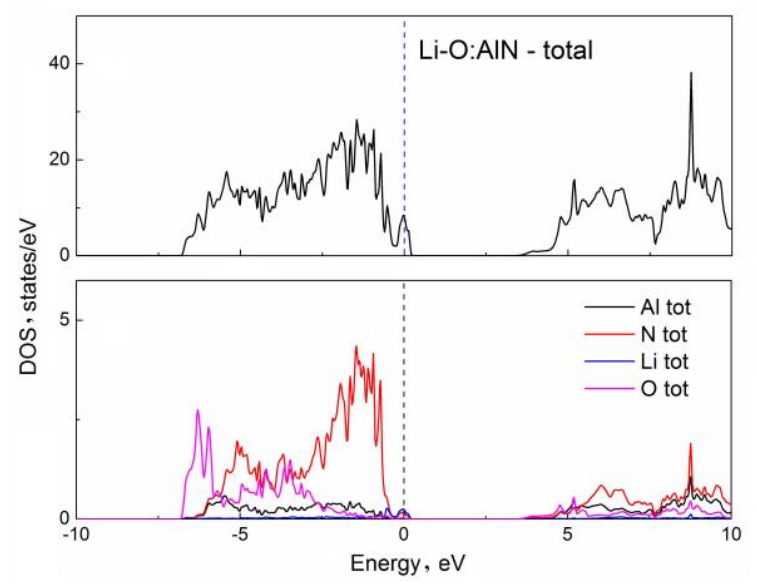

Fig. 3. DOS for Li,O-codoped AlN: Total DOS and Partial DOS. The dashed line denotes the Fermi level

It is also observed that a noticeable shift along the energy scale appears with extra $\mathrm{O}$ insertion, extra states are found in the lower energy levels induced. The change of patterns with given compositions can be mainly attributed to variations in the doping configuration. As mentioned above, charge redistribution effects made the cation and anion bonding in principle ionically polarized. The degree of s-p hybridization increases and the anisotropy becomes larger as the atomic number increases. As seen in the density of states, in addition to distribution of the total states moved towards lower energies, the splitting of states around the Fermi level gradually becomes obvious going from Li to their heavier congeners doped AlN.

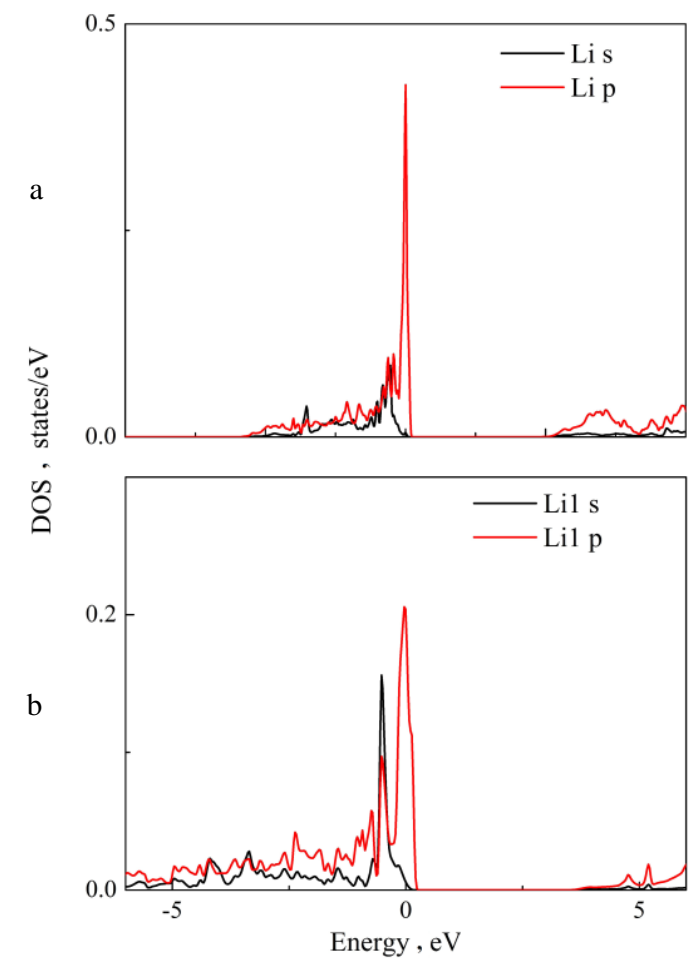

Fig. 4. DOS projected on $\mathrm{Li}$ atoms in $a \mathrm{Li}$-doped $\mathrm{AlN}$ and $b \mathrm{Li}$,Ocodoped AlN

In $\mathrm{Li}$ doped $\mathrm{AlN}, \mathrm{Li}$ atoms are positively charged. Strong overlap of the Li $2 \mathrm{~s}, 2 \mathrm{p}$ states with the $\mathrm{N}$ electronic states will simulate an ionic-like charge transfer. The electrons compensating the holes induced by Li would be in anion $(\mathrm{N}, \mathrm{O}) 2 \mathrm{p}$ bands. Due to the small radius, $\mathrm{Li}^{+}$ cations can move relatively independently [21] so that the inserted $\mathrm{Li}$ atoms are able to donate electron density towards the nearest ions. The electron donation from the doped Li mainly flows toward the $\mathrm{O}^{-}$ions and partly to the $\mathrm{N}^{-}$ions around in codoped supercells. Structural analysis highlights that the codoping leads to charge transfer and weakening of Al-N bonds along with smaller overlap of electronic states. The doped AlN compounds have turned into p-type semiconductors according to the calculated 
band gaps. A negative relationship can be observed between the doped Li impurities and the band gap. The codoping of $\mathrm{Li}$ atom with extra $\mathrm{O}$ yields a significantly narrower band gap compared to the monoclinic doping.

\section{CONCLUSIONS}

The calculated electronic DOS of AIN crystals with Li-doping and Li,O-codoping demonstrated that the doped AlN still exhibits semiconductor nature with p-type character. The codoping has a general smooth effect on the profile. The top of the valence band is flat with large effective masses for localized states with anion $p$ character in $\mathrm{Li}$-doped AlN. It is possible to tune the bandgap of AIN by adjusting doping configuration. According to calculations of formation energy, codoping with $\mathrm{O}$ makes the intercalation of $\mathrm{Li}$ in tetrahedral positions of $\mathrm{AlN}$ energetically favorable regardless of Al-rich or N-rich growth conditions. Both $\mathrm{Li}$ mono-doping and codoping with $\mathrm{O}$ have provided an interesting way to manipulate the corresponding properties of wurtzite AlN crystals.

\section{Acknowledgments}

The authors gratefully acknowledge the financial support from the National Natural Science Foundation of China (Grant No. 11447029, 61440028 and 61136001) and the Science \& Technology Bureau of Shenzhen (Grant No. 20160520174438578).

\section{REFERENCES}

1. Taniyasu, Y., Kasu, M. Origin of Exciton Emissions from an Aln P-N Junction Light-Emitting Diode Applied Physics Letters 98 (13) 2011: pp. 131910.1-131910.3.

https://doi.org/10.1063/1.3574025

2. Wu, H.L., Zheng, R.S., Liu, W., Meng, S., Huang, J.Y. C and Si Codoping Method for P-type AlN Journal of Applied Physics 108 (5) 2010: pp. 053715.1-053715.4. https://doi.org/10.1063/1.3475708

3. Zhang, Y., Liu, W., Niu, H.B. Native defect properties and p-type doping efficiency in group-IIA doped wurtzite AIN Physical Review B 77 (3) 2008: pp. 035201.1-035201.5. https://doi.org/10.1103/PhysRevB.77.035201

4. Ishikawa, R., Lupini, A.R., Oba, F., Findlay, S.D., Shibata, N., Taniguchi, T., Watanabe, K., Hayashi, H., Sakai, T., Tanaka, I., Ikuhara, Y., Pennycook, S.J. Atomic Structure of Luminescent Centers in High-Efficiency Ce-doped W-AlN Single Crystal Scientific Reports 4 (3) 2014: pp. 3778.1-3778.4. https://doi.org/10.1038/srep03778

5. Sharma, V., Manchanda, P., Sahota, P.K., Skomskiand, R., Kashyap, A. Interatomic Exchange in Mndoped III-V Semiconductors Journal of Magnetism and Magnetic Materials 324 (5) 2012: pp. 786-791. https://doi.org/10.1016/j.jmmm.2011.09.017

6. Xu, L., Wang, L.L., Xiao, W.Z., Xiao, G., Huang, W.Q. A Comparative Study on Magnetism in $\mathrm{Zn}$-doped AlN and GaN from First-principles Journal of Applied Physics 116 (10) 2014: pp. 103908.1-103908.5. https://doi.org/10.1063/1.4895057

7. Wu, H.L., Xu, B.S., Zheng, R.S., Yan, Z. First-principle Studies on Ferromagnetism of Fe-doped AlN Diluted Magnetic Semiconductors Materials Science (Medžiagotyra) 22 (4) 2016: pp. 472-475. https://doi.org/10.5755/j01.ms.22.4.10750
8. Xiao, G., Wang, L.L., Rong, Q.Y., Xu, H.Q., Xiao, W.Z. Half-metallic and Magnetic Properties of AlN Nanosheets Doped with Nonmagnetic Metals: A first-principles Study Computational Materials Science 124 2016: pp. 98-105. https://doi.org/10.1016/j.commatsci.2016.07.024

9. Yan, Z., Wu, H.L., Zheng, R.S. Ferromagnetism in AlkaliMetal-doped AlP: An ab Initio Study Computational Materials Science 99 2015: pp. 16-20. https://doi.org/10.1016/j.commatsci.2014.11.053

10. Karthikeyan, B., Sandeep, C.S.S., Pandiyarajan, T., Venkatesan, P., Philip, R. Optical and Nonlinear Absorption Properties of $\mathrm{Na}$ Doped $\mathrm{ZnO}$ Nanoparticle Dispersions Applied Physics Letters 95 (2) 2009: pp. 023118.1-023118.3. https://doi.org/10.1063/1.3182302

11. Reiss, H., Fuller, C.S., Pietruszkiewicz, A.J. Solubility of Lithium in Doped and Undoped Silicon, Evidence for Compound Formation The Journal of Physical Chemistry 25 (4) 1956: pp. 650-655. https://doi.org/10.1063/1.1743021

12. Ogitsu, T., Schwegler, E., Gygi, F., Galli, G. Melting of Lithium Hydride under Pressure Physical Review Letters 91 (17) 2003: pp. 175502.1-175502.4. https://doi.org/10.1103/PhysRevLett.91.175502

13. Van de Walle, C.G., Neugebauer, J. First-principles calculations for defects and impurities: Applications to IIInitrides Journal of Applied Physics 95 (8) 2004: pp. $3851-3879$. https://doi.org/10.1063/1.1682673

14. Zhao, M., Wang, W.J., Wang, J., Yang, J.W, Hu, W.J., Guo, L.W., Chen, X.L. An Effective and Efficient Approach to p-type AlN by Be2:O Codoping from Firstprinciples Calculations Modern Physics Letters B 30 (20) 2016: pp. $16502577-16502585$.

https://doi.org/10.1142/S0217984916502572

15. Schwarz, K. DFT Calculations of Solids with LAPW and WIEN2k Journal of Solid State Chemistry $176(2)$ 2003: pp. 319-328. https://doi.org/10.1016/S0022-4596(03)00213-5

16. Schwarz, K., Blaha, P., Madsen, G.K.H. Electronic Structure Calculations of Solids using the WIEN2k Package for Material Sciences Computer Physics Communications 147 (1) 2002: pp. $71-76$. https://doi.org/10.1016/S0010-4655(02)00206-0

17. Zurek, E., Jepsen, O., Andersen, O.K. Muffin-Tin Orbital Wannier-Like Functions for Insulators and Metals Chemphyschem 6 (9) 2005: pp. 1934-1942. https://doi.org/10.1002/cphc.200500133

18. Tuomisto, F., Maki, J.M., Rauch, C., Makkonen, I. On the Formation of Vacancy Defects in III-nitride Semiconductors Journal of Crystal Growth 350 (1) 2012: pp. $93-97$. https://doi.org/10.1016/j.jcrysgro.2011.12.031

19. Han, R.L., Yuan, W., Yang, H., Du, X.B., Yan, Y., Jin, H.M. Possible Ferromagnetism in Li, Na and K-doped AlN: A First-principle Study Journal of Magnetism and Magnetic Materials 326 (1) 2013: pp. 45-49. https://doi.org/10.1016/j.jmmm.2012.08.026

20. Hine, N.D.M., Frensch, K., Foulkes, W.M.C., Finnis, M.W. Supercell Size Scaling of Density Functional Theory Formation Energies of Charged Defects Physical Review B 79 (27) 2009: pp. 024112.1-024112.12. https://doi.org/10.1103/PhysRevB.79.024112

21. Salanne, M., Simon, C., Turq, P. A First-Principles Description of Liquid BeF2 and Its Mixtures with LiF: 2. Network Formation in LiF-BeF2 Journal of Physical Chemistry B 110 (23) 2006: pp. $11461-1146$. https://doi.org/10.1021/jp061002u 